\title{
Evaluation of P-glycoprotein expression in pain relevant tissues: understanding translation of efflux from preclinical species to human
}

\author{
Renu Singh Dhanikula ${ }^{1 *}$, Cyrla Hoffert ${ }^{2}$, Rebecca Grant ${ }^{2}$, Denis Projean ${ }^{1}$, Rosemarie \\ Panetta $^{3}$, Dajan $\mathrm{O}^{\prime}$ Donnell ${ }^{2}$, Marie Roumi ${ }^{1}$ \\ ${ }^{1}$ Drug Metabolism and Pharmacokinetics, ${ }^{2}$ Translational Science, ${ }^{3}$ Bioscience, AstraZeneca R\&D Montreal, Montreal, \\ Québec, Canada
}

*Corresponding Author: E-mail: singrenu@yahoo.com; Tel.: +1 (514) 651-3602; Fax: +1-111-111-112

Received: May 25, 2016; Revised: July 18, 2016; Published: September 30, 2016

\begin{abstract}
Various efflux transporters, such as P-glycoprotein (P-gp) are now widely accepted to have profound influence on the disposition of substrates. Nevertheless, there is paucity of information about their expression and functionality in the pain relevant tissues (such as brain, spinal cord and dorsal root ganglia (DRG)) across various species. Therefore, our attempts were directed at evaluating P-gp expression in these tissues to understand its effect on the central nervous system (CNS) disposition. As a means of characterizing the normal tissue distribution of $P$-gp, immunohistochemistry was performed with two antibodies (C219 and H241) directed against different epitopes of MDR1 gene. Notable expression of P-gp was detected in the DRG of Sprague Dawley rat, Beagle Dog, Cynomolgous monkey as well as human. The expression of P-gp was observed in the CNS tissues with evident species differences, the expression of P-gp in human brain and spinal cord was lower than in rats and dogs but relatively comparable to that in monkeys. However, no species related differences were seen in the expression at the DRG level. Doublelabelling using an antibody against a marker of endothelial cells confirmed that P-gp was exclusively localized in capillary endothelial cells. This study highlights the cross species similarities and differences in the expression of P-gp and thus serves as a vital step in understanding the translation of exposure of P-gp substrates to human.
\end{abstract}

\section{Keywords}

Transporter, CNS, Brain, Spinal cord, Dorsal root ganglia, immunohistochemistry

\section{Introduction}

Developing CNS therapeutics has been one of the most challenging areas of drug discovery and development in the pharmaceutical industry. This has been primarily due to the restricted delivery of the drug across the physiological barriers (e.g. the blood brain barrier etc.) and also due to the lack of understanding of the differences in expression of proteins affecting the transport of drugs [1]. Transport of molecules across these barriers is a highly complex phenomenon driven by passive diffusion alone or in concert with efflux or influx transport [2]. $A B C$ efflux transporters such as $p$-glycoprotein (P-gp) are well known to have profound influence on brain concentration of their substrates [3]. Studies on understanding the contribution of P-gp efflux on CNS exposure have been largely conducted in rodents, studies in human 
and monkey have been sparse; as a consequence, there is limited knowledge about species differences in the efflux of drug molecules across the blood-brain barrier. Syvänen et al., have reported that some P-gp substrates were effluxed out of the rodent brain but not by the primate brain [4]. The observed differences in brain exposure of these compounds in rodents vs primates could be attributed to existence of species difference in P-gp function. There have been some reports of species differences in the affinity of P-gp substrates amongst cell lines transfected with P-gp from different species [5-8]. These results indicate species differences in P-gp function despite the high degree of homology in the amino acid content across species. In vivo evidence for species differences in P-gp function has been demonstrated by Cutler et al., who found that higher concentrations of the P-gp inhibitor GF-120918 were needed in guinea pigs than in rats to achieve the same increase in brain concentrations of an undisclosed P-gp substrate [9]. Besides differences in substrate affinity, differences in P-gp expression levels could also result in differences in P-gp transport capacity. To our knowledge, there have been no reports on the differences in expression patterns of P-gp in brain tissues across various species. In addition, functionality and expression of P-gp at blood spinal cord barrier is controversial and influence of P-gp on spinal cord exposure has not been widely studied [10-13]. Because the site of action for some pain indications are located in spinal cord and dorsal root ganglia (DRG), the knowledge gained from the microenvironment of the spinal cord DRG could be potentially harnessed to understand the role of P-gp in manipulating drug exposure to target proteins expressed in these regions and to aid in the translation of efficacy data of compounds from animals to human $[14,15]$. Thus, in this study our attempts were directed at evaluating the expression of P-gp in the brain, spinal cord and DRG of various species. Rat, dog and monkey were selected for evaluation because they are widely used preclinical species. Expression of P-gp in the tissues of the above mentioned species was evaluated using indirect immunohistochemistry and the cellular localization was determined using immunofluorescence and confocal microscopy.

\section{Experimental}

\section{Animal Tissues}

Tissues were collected from naïve male Sprague Dawley rats, male Beagle dogs and male Cynomolgus monkeys. Tissues were collected from at least three different animals for all species evaluated. All procedures were carried out as per the institutional Animal Care and Use Committee as well as Animal Research: reporting of In Vivo Experiments (ARRIVE) guidelines. Briefly, prior to tissue excision dogs were anaesthetised with ketamine, xylamine and euthanyl. The tissues were excised and immediately frozen in liquid nitrogen. Cynomolgus monkey (Macaca fascicularis) weighed between 2-3 kg. One of the three monkeys was naïve, the other two monkeys were given a washout period of 4 weeks prior to tissue excision. For tissue excision, monkeys were anaesthesized by ketamine and sodium pentobarbital prior to being scarified. Tissues were harvested and stored in liquid nitrogen and subsequently stored at $-80{ }^{\circ} \mathrm{C}$. Transverse tissue sections were cut at $14 \mu \mathrm{m}$ using a Microm HM 500 OM cryostat (Microm, Walldorf, Germany) after embedding the tissues in tissue freezing medium (Jung, Nussloch, Germany).

\section{Human Tissues}

Human tissues were obtained from the University of Minnesota as per the institutional ethical guidelines and the Code of Ethics of the World Medical Association (Declaration of Helsinki). Tissues were collected from two male and three female individuals with age ranging between 18-56 years. None of the individual had reported neurological condition. Tissue sectioning was performed as outlined in the previous section. 


\section{Immunohistochemistry}

Expression of P-gp protein was mapped using indirect immunohistochemistry. All the experiments were conducted on histologically well preserved fresh frozen tissues. Two distinct antibodies against P-gp were employed as primary antibodies, namely, C219 (mouse monoclonal antibody) and H241 (rabbit anti-human polyclonocal antibody) (Calbiochem, San Diego, CA, USA and Santa Cruz Biotechnologies, Dallas, Texas, USA, respectively). Frozen sections were thawed for 5 min followed by fixation with ice cold acetone for 5 min. Tissue sections were washed 3 times in $0.1 \mathrm{M}$ TBS (tris buffered saline) and then quenched with $0.5 \%$ hydrogen peroxide. This was followed by washing steps with $0.1 \mathrm{M} \mathrm{TBS}$, then sections were subsequently blocked with blocking buffer ( $5 \%$ normal goat serum) for $1.5 \mathrm{~h}$. The blocking medium was removed and sections were incubated overnight with appropriately diluted primary antibody. As a negative control, the primary antibodies were replaced by serum to determine the degree of artifactual labelling by the secondary antibodies (biotinalyted goat anti-mouse IgG or biotinalyted goat anti-rabbit IgG, Vector Laboratories, Burlingame, CA, United States). Additionally, the primary antibody was incubated with blocking peptide to delineate non-specific labelling by the primary antibody. Sections were then rinsed three times with $0.1 \%$ BSA (bovine serum albumin) in $0.1 \mathrm{M} \mathrm{TBS}$, and then incubated for 30 min with avidin-biotinylated horseradish peroxidase complex (Vectastain, Vector laboratories, Burlingame, CA, United States). Diaminobenzidine (Immpact DAB, Vector laboratories, Burlingame, CA, United States) was used as a chromogen. After the treatment, sections were washed with distilled water, dehydrated with ethanol (50, 70, 95 and $100 \%$ ) and defatted in xylene and mounted with Permount mounting medium. Bright field images were captured using a Leica DM4000B microscope (Leica Microsystems, Wetzlar, Germany).

\section{Immunofluorescence and confocal microscopy}

Cellular localization of P-gp was assessed by immunofluorescent co-localization with the C219 antibody and an antibody raised against endothelial cell marker, Von-Willebrand factor (VWF, rabbit polyclonocal antibody, Abcam, Cambridge, United Kingdom). For double labelling, tissue sections were allowed to air dry for approximately $5 \mathrm{~min}$. They were then fixed with ice cold acetone $\left(4^{\circ} \mathrm{C}\right)$ for $5 \mathrm{~min}$. After being rinsed with $0.1 \mathrm{M}$ TBS thrice, slides were incubated with $0.5 \%$ hydrogen peroxide for $10 \mathrm{~min}$ at room temperature. Slides were rinsed again and incubated with blocking buffer ( $5 \%$ normal goat serum) for $1.5 \mathrm{~h}$ at room temperature. C219, P-gp primary antibody was added to each slide followed by overnight incubation at $4{ }^{\circ} \mathrm{C}$. The next day, slides were washed slides with $0.1 \% \mathrm{BSA}$ in $0.1 \mathrm{M}$ TBS and then incubated with secondary antibody (Alexa 488 goat anti-mouse, Vector Laboratories, Burlingame, CA, United States) for C219 for $2 \mathrm{~h}$. Subsequently, all sections were rinsed with $0.1 \%$ BSA in $0.1 \mathrm{M} \mathrm{TBS}$, blocked with blocking buffer (5\% normal donkey serum) for Von Willebrand factor (VWF) antibody for $1.5 \mathrm{~h}$ at room temperature and then incubated with VWF primary antibody for $4.5 \mathrm{~h}$ at $4{ }^{\circ} \mathrm{C}$. Slides were rinsed with $0.1 \%$ BSA in $0.1 \mathrm{M}$ TBS and subsequently incubated with secondary antibody for VWF (Cy-3 conjugated donkey anti-rabbit, Jackson Laboratories, West Grove, PA, United States) for $45 \mathrm{~min}$. Slides were coversliped using Aqua polymount after being rinsed with $0.1 \%$ BSA in $0.1 \mathrm{M}$ TBS. Fluorescent signals from double labelled sections were detected and recorded using a confocal microscope (Zeiss confocal laser scanning TCS SPE microscope, Carl Zeiss Microscopy GmbH, Jena, Germany). Post-acquisition all fluorescent images were analysed by ImageJ analysis software for intensity and area of P-gp labelling/expression. 


\section{Results and Discussion}

The published literature on the expression of P-gp in the CNS largely focuses on its expression in the brain tissue; consequently, there is a paucity of information on its expression in other CNS tissues. In particular, the reports on the expression of this trans-membrane efflux pump in the spinal cord and DRG have been sparse. The lack of understanding of species differences in expression and functionality of transporters can serve as a bottleneck in the translation of drug disposition from preclinical species to human, particularly, for compounds with the site of action in CNS [16]. Therefore, in an attempt to examine species differences in P-gp expression in various pain relevant tissues, we have looked at the detailed micro-anatomic localization of P-gp in the normal brain, spinal cord and DRG tissues of rat, dog, monkey and human by using immunohistochemistry, immunofluorescence and confocal microscopy. To the best of our knowledge, this is the first report in the literature wherein expression of P-gp has been shown in the DRG and spinal cord across several species. Nonetheless, expression of P-gp in various tissues has been examined previously by immunohistochemistry and several monoclonal antibodies have been described in the literature for immunohistochemical detection of its internal or external epitopes [17-19]. In this study, antibodies recognizing two distinct epitopes were used to minimize false-positive results due to binding of antibody to proteins with similar epitopes as well as minimize false-negative results from the failure of antibody binding due to inadequate tissue processing. We have selected C219 as one of the antibodies, because it is one of the most widely used antibody for P-gp immunodetection in both research and diagnosis $[20,21]$. It recognizes amino acid sequence VQEALD of the cytoplasmic region of the protein, which is conserved in all cytosolic domains of P-gp from rodents to human [20, 22, 23]. H-241, a polyclonal antibody that recognizes amino acids 1040-1280 at the P-gp C-terminal cytoplasmic domain of human, mouse and rat origin was another antibody selected for this study. The specificity of both antibodies has been shown by manufacturers using western blotting, immunoprecipitation and immunohistochemistry. We have additionally validated the specificity of these antibodies by testing in multidrug resistant cell lines (MDR1-MDCK and DLD-1).

A brown immunoreactive labelling (Figure 1) representing presence of P-gp protein was formed by reaction of diaminobenzidine chromogen with horseradish peroxidase complexed to $\mathrm{C} 219$ and H241 antibodies. The pattern of brown staining in the tissue sections was considered to be reflective of presence of P-gp protein in tissues. Overall expression of P-gp was found to be similar with both antibodies in all species and tissues examined, suggesting absence of both false-positives and negatives (data not shown). In negative controls, wherein primary antibody was omitted or when the blocking peptide was used, P-gp labelling was not observed (data not shown). This finding together with similar labelling by C219 and H241 antibodies, indicate absence of non-P-gp specific imunnolabelling. For the simplicity of presentation we have reported results of only C219 antibody in the figures. The immunohistographs of the DRGs and spinal cord sections showed prominent labelling of P-gp protein in the microvessels (Figure 1). The level of expression of P-gp in DRG appeared to be consistent across rat, dog, monkey and human as seen in Figures 1 a to $1 \mathrm{~d}$. Immunoreactivity in the spinal cord was uniformly distributed in all the lamellas of the spinal cord with no specific expression pattern (Figures $2 \mathrm{a}$ to $2 \mathrm{~d}$ ). Spinal cord sections from thoracic, lumbar and sacral regions were examined and regional differences were not detected (data not shown). However, grey matter of the spinal cord had relatively higher P-gp expression compared to the white matter. We have also assessed P-gp expression in the brain of rat, dog, monkey and human. P-gp was expressed in the cortical tissues of all species (Figures $3 a$ to $3 d$ ) as has been reported previously [24, 25]. The localization of P-gp in the brain is controversial with some studies suggesting endothelial expression [24, 25], while others reporting expression in the astrocytes or glial cells [26-28]. Nonetheless, even by using antibodies against 
distinct P-gp epitopes (C219 and H241), immunoreactivity was exclusively detected in capillary endothelial cells; neuronal expression of P-gp in the brain tissue was not observed in the species evaluated in the present study. Distribution of P-gp in the microvessels was also confirmed by immunofluorescence technique (Figure 3). The observation regarding endothelial localization of P-gp could be due probably due to the fact that the tissues used in the present study were from healthy animals and non-diseased human donors with no reported neurological conditions.
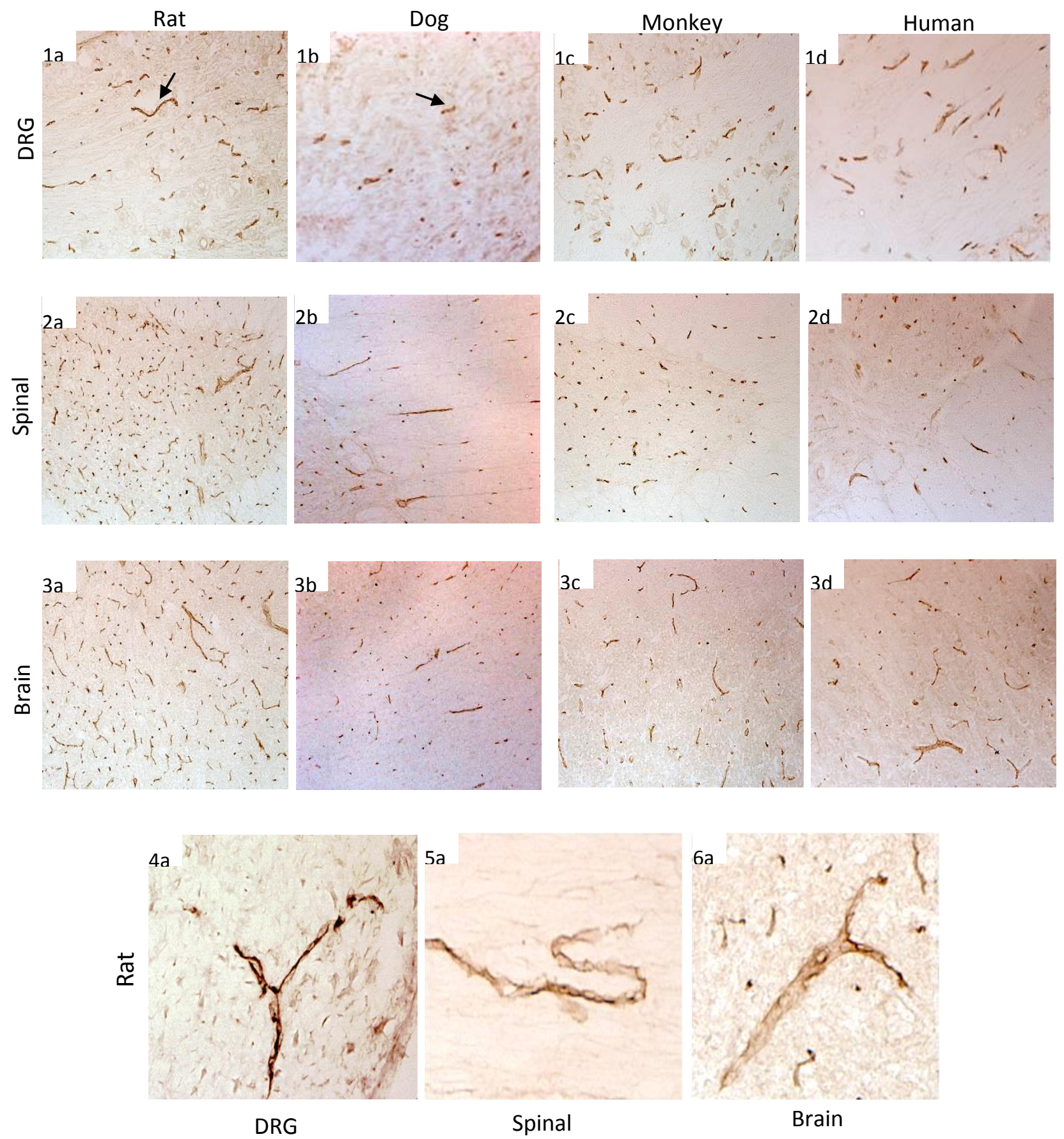

Figure 1. Distribution of P-gp in Sprague Dawley rat (1a, 2a, 3a, 4a, 5a, 6a), Beagle dog (1b, 2b, 3b), Cynomolgus monkey $(1 c, 2 c, 3 c)$ and human $(1 d, 2 d, 3 d)$ dorsal root ganglia, spinal cord and brain cortex as determined by immunohistochemical staining. C219 was used a primary antibody and biotinylated goat antimouse IgG was used as a secondary antibody. Images 1 a to $3 \mathrm{~d}$ were acquired at 10X, while 4 a to 6a were captured at 40X. Data are representative of images captured for each species and tissue $(n=3)$. Arrows indicate P-gp labelling. 
Immunofluorescence and confocal microscopy were done to understand the micro-anatomical localization of P-gp in the tissues, mainly localization in endothelial cells was evaluated. For this purpose, endothelial cells were labelled by VWF antibody (Cy-3 fluorophore) and P-gp by C219 antibody (Alexa 488 fluorophore) and images were acquired at wavelengths specific to both fluorophores. Rich distribution of P-gp was observed in the microvessels of various CNS tissues by immunofluorescence imaging. As seen in Figures 2 and 3, the green fluorescent signal from the labelling of P-gp (C219) and red fluorescent signal from endothelial cells labelling (VWF) overlayed remarkably well, suggesting largely endothelial expression of P-gp. The analysis of the acquired images indicated approximately $95 \%$ of all endothelial cells were labelled by P-gp antibodies in both brain and spinal cord. We did not observe any marked changes in the microvessel localization of P-gp with species. However, as evidenced by intense labelling in the immunohistographs, the highest level of P-gp expression in the microvessels was observed in the DRG tissues of all species examined (Figures $2 c, 2 f \& 2 i)$.
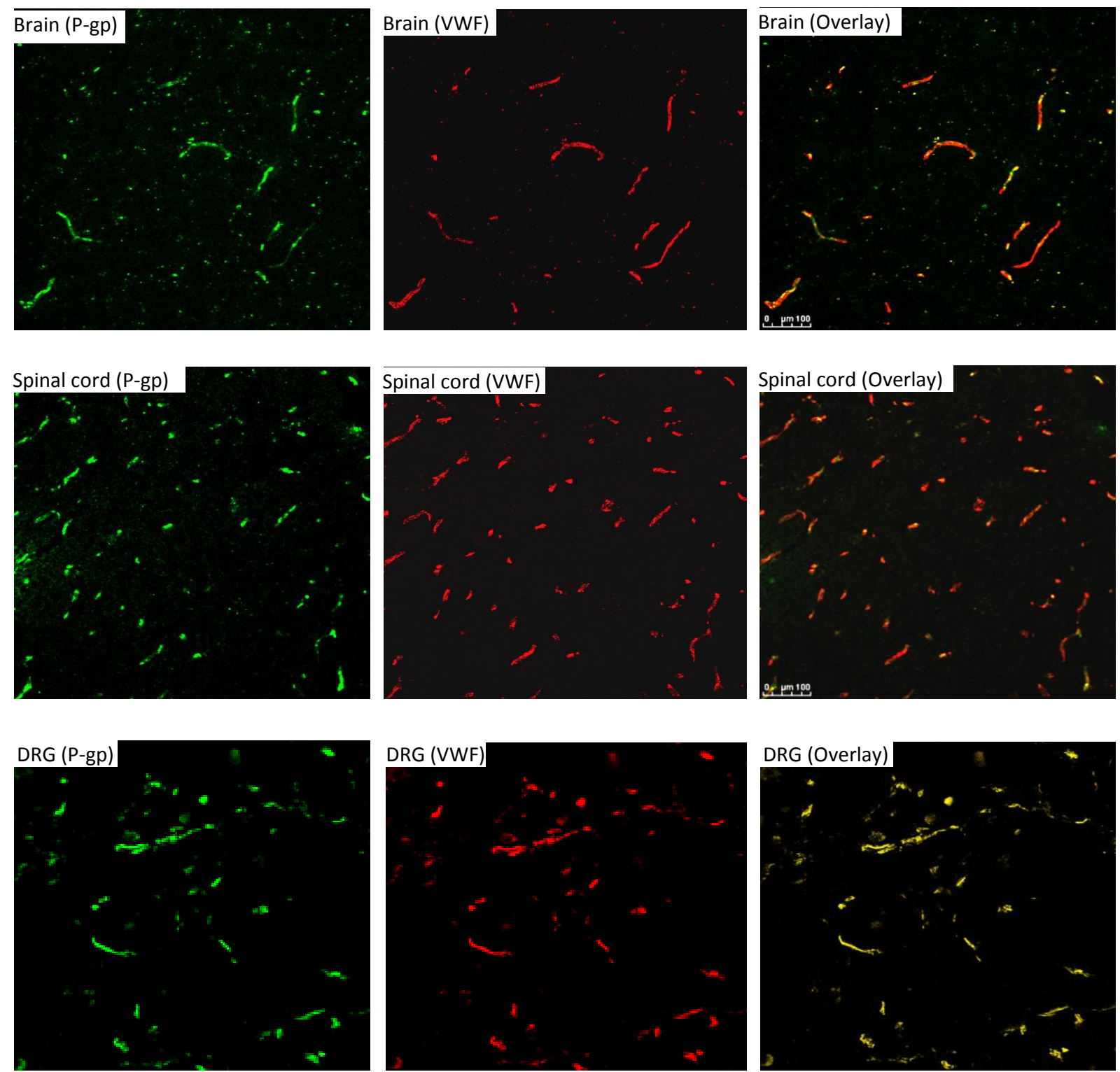

Figure 2. Confocal microscope images of fresh frozen Human tissues labelled with C219 and VWF antibodies for P-gp and endothelial cells, respectively. Cy-3 goat anti-mouse was used a secondary antibody for P-gp and Alexa-Fluor 488 goat anti-rabbit for endothelial cells. The green panel (left) is indicative of P-gp expression in the tissues, while the red panel (middle) displays endothelial cell expression. The right panel is the overlay of both red and green signals demonstrating co-localization. Images were acquired at $10 \mathrm{x}$. Data are representative of images captured for each tissue $(n=3)$. 
Regional differences were observed within the brain cortex in all species, with the grey matter exhibiting higher protein levels relative to the white matter. These regional differences are considered to be primarily due the extent of vascularisation of these regions as can be seen from Figures 2 and 3 . The intensity and area of labelling in the CNS tissues evidently decreased from rat to human. For instance, the expression of P-gp in the brain cortex was relatively higher in rat than in dog followed by monkey and human. It is important to note that P-gp expression in human and monkey was comparable. A similar trend was observed in the spinal cord expression of P-gp. Interestingly, there were no species differences in the P-gp expression in the DRG.
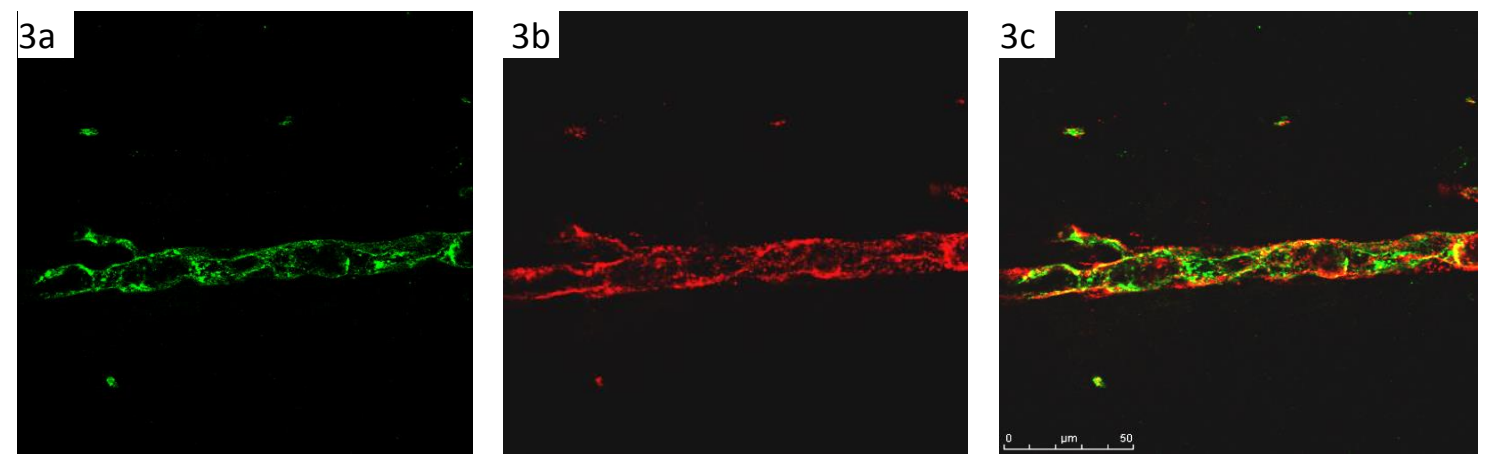

Figure 3. Fluorescence images of fresh frozen Human tissues labelled with C219 and VWF antibodies, acquired with a confocal microscope at 40x. The green signal is indicative of P-gp labelling in the tissues, while the red signal is for endothelial cell expression. The overlay of the two signals is shown in the right panel.

\section{Conclusions}

In this study we have examined the expression and distribution of P-gp in the pain relevant tissues of species widely employed in preclinical research namely Sprague Dawley rat, Beagle Dog, Cynomolgous monkey and have compared to that in human. We have also shown that P-gp is expressed in the DRG and spinal cord, and that there are species specific and regional differences in P-gp expression in the brain cortex and spinal cord. The findings of this study serves as a step forward in understanding species differences in transporter expression and may help build our understanding of the translation of drug exposure and efficacy from preclinical species to human.

Acknowledgements: Authors thank Francoise Mennicken for her advice and technical support.

\section{References}

[1] F. Girardin, Dialogues Clin Neurosci. 8 (2006) 311-321.

[2] W. M. Pardridge, NeuroRx. 2 (2005) 3-14.

[3] P. L. Golden, G. M. Pollack, J. Pharm. Sci. 92 (2003) 1739-1753.

[4] S. Syvänen, O. Lindhe, M. Palner, B. R. Kornum, O. Rahman, B. Långström, G. M. Knudsen, M. Hammarlund-Udenaes, Drug Metab. Dispos. 37 (2009) 635-643.

[5] T. Ohe, M. Sato, S. Tanaka, N. Fujino, M. Hata, Y. Shibata, A. Kanatani, T. Fukami, M. Yamazaki, W. M. Pardridge, P. L. Golden, Y. S. Kang and U. Bickel, J. Neurochem. 68 (1997) 1278-1285.

[6] M. Katoh, N. Suzuyama, T. Takeuchi, S. Yoshitomi, S. Asahi, T. Yokoi, J. Pharm. Sci. 95 (2006) 26732683.

[7] C. Q. Xia, G. Xiao, N. Liu, S. Pimprale, L. Fox, C. J. Patten, C. L. Crespi, G. Miwa, L. S. Gan, Mol. Pharm. 3 (2006) 78-86. 
[8] S. Baltes, A. M. Gastens, M. Fedrowitz, H. Potschka, V. Kaever, W. Lo"scher, Neuropharmacology 52 (2007) 333-346.

[9] L. Cutler, C. Howes, N. J. Deeks, T. L. Buck, P. Jeffrey, J. Pharm. Sci. 95 (2006) 1944-1953.

[10] N.J. Abbott, A.A. Patabendige, D.E. Dolman, S.R. Yusof, D.J. Begley, Neurobiol. Dis 37 (2010) 13-25.

[11] A.D. Wong, M. Ye, A.F. Levy, J.D. Rothstein, D.E. Bergles, P.C. Searson, Front Neuroeng. 6 (2013) 7.

[12] T. Ashraf, A. Kao, R. Bendayan, Adv. Pharmacol. 71 (2014) 45-111.

[13] W. Loscher, H. Potschka, NeuroRx 2 (2005) 86-98.

[14] E. C. deLange, P. G. Ravenstijn, D. Groenendaal, T.J. van Steeg, AAPS J. 7 (2005) E532-E543.

[15] M. Danhof, E. C. de Lange, O. E. Della Pasqua, B. A. Ploeger, R. A. Voskuyl, Trends Pharmacol. Sci. 29 (2008) 186-191.

[16] X. Chu, K. Bleasby, R. Evers, Expert Opin Drug Metab Toxicol. 9 (2013) 237-252.

[17] P. M. Chaudhary, E. B. Mechetner, I. B. Roninson, Blood 80 (1992) 2735-2739.

[18] A. H. Schinkel, R. J. Arceci, J. J. Smit, E. Wagenaar, F. Baas, M. Dollé, T. Tsuruo, E.B. Mechetner, I. B. Roninson, P. Borst, Int J. Cancer. 55 (1993) 478-484.

[19] T. Grogan, W. Dalton, J. Rybski, C. Spier, P. Meltzer, L. Richter, M. Gleason, J. Pindur, A. Cline, R. And Scheper, Lab Invest. 63 (1990) 815-824.

[20] J. M. van Den Elsen, D. A. Kuntz, F. J. Hoedemaeker, D. R. Rose, Proc. Natl. Acad. Sci. USA 96 (1999) 13679-13684.

[21] E. Okochi, T. Iwahashi, T. Tsuruo, Leukemia 11 (1997) 1119-1123.

[22] E. Georges, G. Bradley, J. Gariepy, V. Ling, Proc. Natl. Acad. Sci. USA. 87 (1990) 152-156.

[23] L. Jetté, B. Têtu and R. Béliveau, Biochim Biophys Acta. 1150 (1993) 147-154.

[24] Y. Matsuoka, M. Okazaki, Y. Kitamura, T. Taniguchi, J. Neurobiology 39 (1999) 383-392.

[25] I. Sugawara, H. Hamada, T. Tsuruo, S. Mori, Jpn. J. Cancer Res. 81 (1990) 727-730.

[26] W.M. Pardridge, P.L. Golden, Y.S. Kang, U. Bickel, J. Neurochem. 68 (1997) 1278-1285.

[27] X. Decleves, A. Regina, J. L. Laplanche, F. Roux, B. Boval, J. M. Launay, J. M. Scherrmann, J. Neurosci. Res. 60 (2000) 594-601.

[28] G. Lee, L. Schlichter, M. Bendayan,R. Bendayan, J. Pharmacol. Exp. Ther. 299 (2001) 204-212.

C2016 by the authors; licensee IAPC, Zagreb, Croatia. This article is an open-access article distributed under the terms and conditions of the Creative Commons Attribution license (http://creativecommons.org/licenses/by/3.0/) (cc)) EY 